Sección Básica / Basic

Ensayo / Essay

\title{
Consideraciones sobre el estado del conocimiento de la diversidad de Coleoptera (Arthropoda: Insecta) en Colombia
}

\author{
Considerations on the status of the knowledge of coleopteran diversity \\ (Arthropoda: Insecta) in Colombia
}

\begin{abstract}
(iD JENNIFER C. GIRÓN ${ }^{1,2^{*}}$; iD GERMÁN AMAT-GARCÍA †; (iD JUAN PABLO BOTERO $^{4}$; ID JULIANA CARDONA-DUQUE ${ }^{5}$; (D) JULIÁN CLAVIJO-BUSTOS ${ }^{6}$; (D) CAMILA DÍAZ-DURÁN 7; iD JORGE HUMBERTO GARCÍA CONCHA ${ }^{3}$; (iD KIMBERLY GARCÍA ${ }^{4}$; (iD WILBER LÓPEZ MURCIA ${ }^{3}$; (iD ANDRÉS LUGO DE LA HORTÚA ${ }^{3}$; iD JHON CESAR NEITA MORENO ${ }^{8}$; iD JOSÉ M. RAMÍREZ-SALAMANCA ${ }^{9}$; iD CARLOS TABOADA-VERONA ${ }^{10}$; (iD DIEGO UCHIMA TABORDA ${ }^{11}$; iD ALEJANDRA VIASUS-BASTIDAS ${ }^{5}$
\end{abstract}

\begin{abstract}
${ }^{1}$ Purdue University, West Lafayette, Indiana, EEUU, entiminae@gmail.com ${ }^{2}$ Natural Science Research Laboratory, Museum of Texas Tech University, Lubbock, Texas, EEUU. ${ }^{3}$ Universidad Nacional de Colombia, Instituto de Ciencias Naturales, Bogotá, Colombia, jhgarciac@unal.edu.co, wlopezm@unal.edu.co, anluogho@unal.edu.co ${ }^{4}$ Universidade de São Paulo, Museu de Zoologia da, São Paulo, Brasil, jp_bot@yahoo.com, kimberly.pg@gmail.com ${ }^{5}$ Universidad CES, Facultad de Ciencias y Biotecnología, Medellín, Colombia; jcardonad@ces.edu.co, viasus.maria@uces.edu.co ${ }^{6}$ Universidad del Tolima, Facultad de Ciencias, Ibagué, Tolima.; jclavijob@ut.edu.co ${ }^{7}$ Universidad de La Salle, Departamento de Ciencias Básicas, Bogotá, Colombia; diazmaca29@gmail.com ${ }^{8}$ Instituto de Investigación de Recursos Biológicos Alexander von Humboldt, Colecciones Biológicas, Villa de Leyva, Boyacá, Colombia; jneita@humboldt. org.co ${ }^{9}$ Universidad Nacional de Cuyo, Instituto Argentino de Investigaciones de las Zonas Áridas (IADIZA), Mendoza, Argentina; areisus@gmail.com ${ }^{10}$ Universidad de Sucre, Grupo Evolución y Sistemática Tropical, Sincelejo, Colombia.; carlostaboadaverona@gmail.com ${ }^{11}$ Universidad de Antioquia, Grupo de Entomología Universidad de Antioquia (GEUA), Instituto de Biología, Medellín, Colombia; alejandro.uchima@udea.edu.co.
\end{abstract}

\section{Autor para correspondencia}

Jennifer C. Girón Purdue University, West Lafayette, Indiana, EEUU y Natural Science Research Laboratory, Museum of Texas Tech University, Lubbock, Texas, EEUU; entiminae@gmail.com.

\section{Citación sugerida}

GIRÓN, J. C.; AMAT-GARCÍA, G.; BOTERO, J. P.; CARDONA-DUQUE, J.; CLAVIJO-BUSTOS, J.; DIÁZ-DURÂN, C.; GARCÍA CONCHA, J. H.; GARCÍA, K.; LÓPEZ MURCIA, W.; LUGO DE LA HORTÚA, A.; NEITA MORENO, J. C.; RAMÍREZ-SALAMANCA, J. M.; TABOADA-VERONA, C.; UCHIMA TABORDA, D.; VIASUS-BASTIDAS, A. 2021. Consideraciones sobre el estado del conocimiento de la diversidad de Coleoptera (Arthropoda: Insecta) en Colombia. Revista Colombiana de Entomología 47 (2): e10717. https://doi. org/10.25100/socolen.v47i2.10717

Recibido: 04-Dic-2020

Aceptado: 26-May-2021

Publicado: 07-Ago-2021

Revista Colombiana de Entomología

ISSN (Print): 0120-0488

ISSN (On Line): 2665-4385

https://revistacolombianaentomologia.univalle.edu.co

Open access

(c) (i) (2) (2) BY-NC-SA 4.0

Publishers: Sociedad Colombiana de Entomología SOCOLEN (Bogotá, D. C., Colombia) https://www.socolen.org.co

Universidad del Valle (Cali, Colombia)

https://www.univalle.edu.co

(C) 2021 Sociedad Colombiana de Entomología SOCOLEN y Universidad del Valle - Univalle
Resumen: Coleoptera es uno de los órdenes más diversos en el planeta y Colombia es uno de los países con mayor biodiversidad. La combinación de estos dos factores determina indiscutiblemente una elevada concentración de especies, cuyas consecuencias sólo se conocen parcialmente en el país. En este trabajo se presenta un ensayo sobre la diversidad de coleópteros en Colombia, resaltando el papel de las colecciones entomológicas y los vacíos de información a la hora de abordar la coleóptero-fauna nacional. Esta contribución constituye un punto de partida para que los coleopterólogos de Colombia comencemos a trabajar colectivamente hacia un conocimiento más amplio y menos fragmentado sobre la historia natural, sistemática, ecología y biogeografía del grupo.

Palabras clave: coleópteros; región neotropical; colecciones biológicas; colecciones entomológicas.

Abstract: Coleoptera is one of the most diverse orders on the planet, and Colombia one of the most biodiverse countries. The combination of these two factors determines with no discussion a high concentration of species, with only partially known consequences in the country. In this work we describe the current state of knowledge about the diversity of coleopterans in Colombia, highlighting the role of entomological collections and the knowledge gaps to tackle the national beetle fauna. This contribution is a starting point for coleopterologists of Colombia to begin working together towards a broader and less fragmented knowledge of the natural history, systematics, ecology, and biogeography of the group.

Key words: beetles; Neotropical region; biological collections; entomological collections.

\section{Introducción}

El orden Coleoptera, cuyos integrantes son conocidos popularmente en Colombia como cucarrones o escarabajos, es un grupo de distribución cosmopolita, conformado actualmente por alrededor de 400 mil especies descritas; constituye el grupo animal más diverso del planeta, representando cerca del 25\% de todos los seres vivos y 35\% de los insectos (Grimaldi y Engel 2005; Rafael et al. 2012; Eggleton 2020); el estimado más reciente indica que el número potencial de especies de Coleoptera en el mundo esté entre 1.700 .000 y 2.100 .000 especies (García-Robledo et al. 2020). Los coleópteros tienen gran importancia como proveedores de servicios ecosistémicos, pues desempeñan múltiples funciones en una amplia variedad de ecosistemas: pueden ser depredadores (por ejemplo, Carabidae, Staphylinidae, 
Dytiscidae), fitófagos (por ejemplo, Buprestidae, Cerambycidae, Chrysomelidae), polinizadores (por ejemplo, Curculionidae, Scarabaeidae), o saprófagos (ejemplos: Dermestidae, Histeridae, Silphidae); gracias a esta diversidad de hábitos algunos coleópteros se usan como biocontroladores, o son útiles en investigaciones forenses, o pueden considerarse bioindicadores, o poseen importancia médica o económica, incluyendo algunas especies que se consideran comestibles (Marshall 2018). Actualmente, el orden está compuesto por aproximadamente 180 familias existentes y cerca de 30 familias fósiles, divididas en cuatro subordenes: Archostemata, Myxophaga, Adephaga y Polyphaga (Marshall 2018). Éste último suborden contiene más del $90 \%$ de las especies descritas (Marshall 2018).

Colombia es uno de los 12 países con mayor diversidad biológica del mundo; con apenas el $0,7 \%$ de la superficie continental mundial, concentra cerca del $10 \%$ de la biodiversidad del planeta (Mittermeier y Goettsch 1997; Colciencias 2016), debido principalmente a su posición geográfica en la región neotropical, a los procesos orogénicos de los Andes y al ser una zona de transición en las Américas (Romero et al. 2008; Etter y van Wyngaarden 2000). Aunque se desconocen los patrones de distribución de esta diversidad al interior del país (es decir, si algunas regiones son realmente más ricas que otras), se reconoce que nuestro conocimiento de la biodiversidad en Colombia está sesgado (Arbeláez-Cortés 2013). Existe una tendencia marcada a tener un mayor número de registros de especies en la región Andina, mientras que otras regiones del país como Amazonía, Caribe, Insular, Orinoquía y Pacífico contienen pocos registros o, en ocasiones, estos son inexistentes (por ejemplo, Moreno et al. 2016). Esta escasez regional de registros coincide en varios grupos biológicos incluyendo Coleoptera. Por otra parte, pese a expediciones universitarias y a los esfuerzos recientes a través de las "Expediciones BIO", Colombia continúa representando un enorme vacío de conocimiento para la coleóptero-fauna de la región Neotropical, sobre todo si se compara con otros países donde ya existen proyectos específicos para el levantamiento de la fauna local, como es el caso de países como Brasil (33.210 especies registradas, Monné y Costa 2020) o los listados nacionales que se están produciendo en Perú (por ejemplo, Monné y Chaboo 2015; ver más referencias en https:/www.researchgate.net/ project/Beetles-Coleoptera-of-Peru). Aquí se presenta un panorama general del estado del conocimiento de la diversidad de los Coleoptera en Colombia, indicando algunas tendencias generales en la información disponible.

Estudio de los Coleoptera en Colombia. Las investigaciones con coleópteros en Colombia se desarrollan principalmente desde las universidades en diversos temas como inventarios locales, faunística y distribución, taxonomía y sistemática, ecología, interacciones con otros organismos, bioindicadores, importancia económica, conservación, especies amenazadas, servicios ecosistémicos, entre otros (por ejemplo, Castillo-Velandia et al. 2020). No obstante, el estado del conocimiento de muchos grupos de Coleoptera en Colombia es escaso o incipiente (Deloya y Gasca-Álvarez 2018). En términos de diversidad general del orden, el único listado de especies para el país fue publicado por Blackwelder (1944-1957). En dicho listado aparecen aproximadamente 6.170 especies registradas, agrupadas en 114 familias; sin embargo, se estima que podrían existir cerca de 7.000 especies de coleópteros en Colombia (SiB Colombia 2020).
Existen listados de especies, relativamente recientes, para algunas familias y subfamilias en Colombia: Carabidae (Martínez 2005), Cerambycidae (Martínez 2000), Cerambycidae: Parandrinae (Cardona-Duque et al. 2010), Chrysomelidae: Cassidinae (Borowiec y Swiętojańska 2015), Cicindelidae (Vitolo y Pearson 2003), Elmidae (González-Córdoba et al. 2002), Hybosoridae (Ocampo y Ballerio 2006), Hydrophilidae (González-Rodríguez et al. 2017), Melolonthidae: Melolonthinae, Rutelinae, Dynastinae y Cetoniinae (Neita-Moreno y Gaigl 2008), Melolonthidae: Dynastinae (Gasca-Álvarez y Amat-García 2010), Passalidae (Amat-García et al. 2004), Scarabaeidae: Aphodiinae (Pardo-Locarno y Schoolmeesters 2019), Scarabaeidae: Scarabaeinae (Medina et al. 2001), Silphidae (Bonilla et al. 2016), Staphylinidae (Newton et al. 2005) y Trogidae (Scholtz 1990). Además, existen listados de géneros con número de especies para Curculionidae (Girón y Cardona-Duque 2018) y Elateridae (Aguirre-Tapiero 2009). Estos listados, junto con actualizaciones disponibles (por ejemplo, Botero 2018; Jiménez-Ferbans et al. 2018) acumulan 5.044 especies en sólo 20 familias.

Cada vez que se hacen revisiones faunísticas y taxonómicas, estudiando colecciones colombianas, se hacen nuevos registros y/o se descubren nuevas especies (por ejemplo, Howden 1976, Cardona-Duque et al. 2010; González-Rodríguez et al. 2017; García et al. 2019; Martínez-Revelo et al. 2020). Además, los números de especies aumentan notablemente cuando se estudian regiones poco exploradas (por ejemplo, Martínez-Revelo et al. 2020; González-Córdoba et al. 2019). Por otro lado, existe una larga lista de familias de Coleoptera cuyo estudio ha comenzado recientemente, o que aún no se estudian en Colombia. Varias de estas familias son grupos altamente diversos como Carabidae, Chrysomelidae y Tenebrionidae, entre otras. Existen además familias que, aunque no son tan numerosas, su representación en el país constituye un vacío de conocimiento para nuestra biodiversidad $\mathrm{y}$, por lo tanto, merecen atención (por ejemplo, Byrrhidae, Ptilidae, Nitidulidae, Leiodidae, Lepiceridae, Scirtidae, entre muchas otras).

En muchos grupos de coleópteros en Colombia son evidentes los altos niveles de endemismo (por ejemplo, Botero 2018; Girón y Cardona-Duque 2018). En 2007 se dieron a conocer las primeras especies de Coleoptera con algún riesgo de extinción; estas especies son en su mayoría endémicas y presentan un alto grado de vulnerabilidad (Amat-García et al. 2007), por lo que requieren esfuerzos de conservación. Evaluar el estado de amenaza de nuestras especies endémicas es apremiante en el contexto actual, pues la tasa de deforestación de los bosques tropicales y el avance de la frontera agrícola y ganadera constituyen las mayores amenazas para los ecosistemas nacionales, especialmente en las regiones de la Amazonía y la Orinoquía, precisamente dos de las áreas menos estudiadas en el país (Moreno et al. 2016).

La importancia de las colecciones biológicas. Las colecciones entomológicas colombianas, como repositorios de nuestra biodiversidad, son fuentes de información esenciales en el desarrollo de la coleopterología en el país. De acuerdo con el Registro Nacional de Colecciones (RNC; http://rnc.humboldt.org.co/wp/) en Colombia se reportan 156 colecciones entomológicas, de las cuales una es exclusivamente coleopterológica (Colección Familia Pardo Locarno, registro No. 87). Al momento, la colección coleopterológica mejor documentada, o al menos cuya información es más accesible, es la 
colección del Instituto Alexander von Humboldt, la cual cuenta con más de 60.000 registros de coleópteros que constituyen más del $80 \%$ de los datos nacionales. Algunas otras colecciones nacionales importantes por su representatividad taxonómica y geográfica son la Colección Entomológica del Instituto de Ciencias Naturales de la Universidad Nacional de Colombia (Bogotá, registro No. 5), el Museo de Entomología de la Universidad del Valle (Cali, registro No. 77) y la colección del Museo Entomológico "Francisco Luis Gallego" (Medellín, registro No. 8). De esta última se han publicado algunos listados de especímenes, por ejemplo para Lampyridae (Ladino 2019) y Lucanidae (Tabares-Duque et al. 2016), entre otros. Los diagnósticos, resultado de curadurías con evaluaciones del estado de salud de las colecciones coleopterológicas en el país, iniciaron con el diagnóstico de la colección de escarabajos coprófagos (Scarabeidae: Scarabaeinae) del Museo Entomológico Francisco Luis Gallego de la Universidad Nacional de Colombia en la sede Medellín (Martínez-Revelo y Medina 2017); se requiere que estas tareas se hagan extensivas por lo menos a las colecciones coleopterológicas en los próximos años. Identificar, curar, organizar y digitalizar los especímenes de colecciones nacionales, incluyendo colecciones locales más pequeñas, constituye un aspecto fundamental que permitiría aunar esfuerzos y avanzar hacia un inventario de la biodiversidad del orden Coleoptera en el país.

Recientemente, gracias a iniciativas nacionales como el $\mathrm{SiB}$ Colombia (https://sibcolombia.net/), los datos que reposan en colecciones colombianas poco a poco se movilizan hacia plataformas en línea, donde pueden ser consultados por un amplio rango de usuarios. Sin embargo, hasta ahora existen relativamente pocos datos sobre coleópteros en estos sistemas, comparados con la gran cantidad de especímenes que reposa en las colecciones. La poca disponibilidad de registros se debe en parte a la falta de apoyo institucional, pues no hay personal en condiciones laborales idóneas (incluyendo falta de capacitación, falta de incentivos académicos y económicos y falta de infraestructura tecnológica, entre otros) para procesar esta información.

Una tarea importante para quienes tienen acceso, o están a cargo de colecciones entomológicas en Colombia, es visibilizar sus fortalezas (por ejemplo, informar qué material se tiene por grupo o por región, si hay material identificado por especialistas, etc.). Disponer de información sobre las colecciones que han sido revisadas para determinados grupos, resulta fundamental para $(i)$ definir qué colecciones necesitan revisiones, (ii) saber qué grupos han sido revisados, (iii) establecer en qué colecciones hay material identificado por expertos, y (iv) determinar qué grupos están mejor representados en colecciones particulares. De esta forma, al tener información organizada, la comunidad coleopterológica colombiana podría ser más eficiente a la hora de encaminar a nuevos estudiantes o dirigir esfuerzos como las giras de especialistas.

Dificultades en el estudio del grupo. Conocer nuestra coleóptero-fauna es una labor difícil, pero a la vez urgente y fundamental. Existen grandes dificultades a la hora de identificar especímenes recolectados en el territorio colombiano, que radican principalmente en la falta, inaccesibilidad, fragmentación o desconocimiento de la literatura especializada sobre la fauna colombiana. La falta de estudios taxonómicos y especialistas nacionales, junto con la escasez de colecciones de referencia con identificaciones confiables (es decir, verificadas por especialistas) en el país, hacen que la tarea de identificar y conocer nuestra fauna sea aún más complicada, especialmente en aquellos grupos que aún no han sido trabajados y necesitan mayor atención. Otro factor que dificulta la identificación es que, en muchos casos, las especies sólo se conocen de las descripciones originales, publicadas aproximadamente entre 1800 y 1900, en las que son comunes localidades como "Nueva Granada" o simplemente "Colombia", información que imposibilita ubicar las localidades tipo y conocer las distribuciones en el territorio nacional. Además, la mayoría del material tipo se encuentra en colecciones extranjeras, principalmente en Europa y Norteamérica, puesto que una gran proporción de trabajos taxonómicos que incluyen material colombiano han sido - y continúan siendo - realizados por investigadores extranjeros. Estas condiciones implican un alto esfuerzo, costo y complicaciones adicionales para quienes emprenden trabajos de revisión taxonómica en Colombia, y es tal vez una de las razones por las cuales se estudian con frecuencia sólo los grupos mejor conocidos. No es la primera vez que estos problemas han sido examinados, sino que se han discutido anteriormente para Coleoptera en Colombia (por ejemplo, Amat y Escobar 2000). En casi 20 años, a pesar de la aparición de más listados por familias, el panorama no ha cambiado significativamente.

A finales de 2020 se conformó el Grupo de Coleopterólogos de Colombia (ColeopCol; https://sites.google.com/ view/coleopcol), haciendo un llamado a los coleopterólogos del país, con el ánimo de compilar y difundir la información disponible sobre los diferentes grupos taxonómicos, y crear un directorio de especialistas que trabajan actualmente con la coleóptero-fauna nacional. El siguiente paso será compilar listados nacionales de especies para los diferentes grupos, y compartirlos a través del portal de datos de SiB Colombia. Entre las tareas a desarrollar a mediano plazo están la producción del catálogo de los coleópteros de Colombia, evaluar el estado de conservación y amenaza de las especies del país, e incentivar y apoyar la sistematización de las colecciones biológicas. Se espera que este colectivo sirva como apoyo a la formación de nuevas generaciones de especialistas nacionales que tanto necesitamos.

\section{Literatura citada}

AGUIRRE-TAPIERO, M. P. 2009. Clave de identificación de géneros conocidos y esperados de Elateridae Leach (Coleoptera: Elateroidea) en Colombia. Boletín del Museo de Entomología de la Universidad del Valle 10 (2): 25-35- http://hdl.handle. net/10893/732

AMAT, G.; ESCOBAR, F. 2000. Capacidad nacional de investigación en sistemática biológica en Colombia y breve reseña del estado actual del conocimiento taxonómico del orden Coleoptera. pp. 137-144. En: Martín-Piera, F.; Morrone, J. J.; Melic, A. (Eds.) Hacia un Proyecto CYTED para el Inventario y Estimación de la Diversidad Entomológica en Iberoamérica: PrIBES-2000. Monografías Tercer Milenio, Vol. 1, SEA, Zaragoza, $326 \mathrm{p}$.

AMAT-GARCÍA, G.; ANDRADE-CORREA, G.; AMAT-GARCÍA, E. 2007. Libro Rojo de los Invertebrados Terrestres de Colombia. Universidad Nacional de Colombia-Conservación Internacional Colombia-Instituto Alexander von Humboldt-Minambiente. 216 pp. http://www.bio-nica.info/Biblioteca/ Amat-Garcia2007LibrRojoInvertebrados.pdf

AMAT-GARCÍA, G.; BLANCO-VARGAS, E.; REYES-CASTILLO, P. 2004. Lista de especies de los escarabajos pasálidos (Coleoptera: Passalidae) de Colombia. Biota Colombiana 5 (2): 
173-182. http://revistas.humboldt.org.co/index.php/biota/article/ view/143

ARBELÁEZ-CORTÉS, E. 2013. Knowledge of Colombian biodiversity: published and indexed. Biodiversity and Conservation, 22 (12): 2875-2906. https://doi.org/10.1007/s10531-013-0560-y

BLACKWELDER, R. E. 1944-1957. Checklist of the coleopterous insects of Mexico, Central America, The West Indies, and South America. Bulletin of the United States National Museum 185 (16): 1-1492. https://www.biodiversitylibrary.org/page/7876356

BONILLA, M.; NAVARRETE-HEREDIA, J. L.; NORIEGA J.A. 2016. Silphidae (Insecta: Coleoptera) de Colombia: Diversidad y Distribución. Boletín de la Sociedad Entomológica Aragonesa 58: 135-152. http://www.sea-socios.com/Boletines/PDF/ Boletin58/135-152BSEA58.pdf

BOROWIEC, L.; ŚWIĘTOJAŃSKA, J. 2015. Checklist of tortoise beetles (Coleoptera, Chrysomelidae, Cassidinae) from Colombia with new data and description of a new species. ZooKeys 2015 (518): 87-127. https://doi.org/10.3897/zookeys.518.9350

BOTERO, J. P. 2018. La familia Cerambycidae (Coleoptera: Chrysomeloidea) en Colombia. pp. 153-169. En: Deloya, C.; Gasca Álvarez, H. J. (Eds). Escarabajos del Neotrópico (Insecta: Coleoptera). S y G editores, México, $264 \mathrm{p}$.

CARDONA-DUQUE, J.; SANTOS-SILVA, A.; WOLFF, M. 2010. Parandrinae (Coleoptera: Cerambycidae) de Colombia. Revista Colombiana de Entomología 36 (1): 135-157. http://www. scielo.org.co/scielo.php?pid=S0120-04882010000100022\&scr $\mathrm{ipt}=$ sci arttext\&tlng $=$ en

CASTILLO-VELANDIA, D. R.; VARGAS-OROZCO, G. A.; VIDAL-GÓMEZ, U.; TIRADO-PINZÓN, H. Y. (Comp.). 2020. Memorias Congreso Sociedad Colombiana de Entomología. 47 Congreso SOCOLEN. Congreso virtual. Sociedad Colombiana de Entomología. 8 y 9 de octubre de 2020, Bogotá, D. C., Colombia. $221 \mathrm{pp}$.

COLCIENCIAS. 2016. Colombia, el segundo país más biodiverso del mundo. https://www.minambiente.gov.co/index.php/noticias-minambiente/4317-colombia-el-segundo-pais-mas-biodiverso-del-mundo-celebra-el-dia-mundial-de-la-biodiversidad [Fecha revisión: 1 diciembre 2020].

DELOYA, C., GASCA-ÁLVAREZ, H. J. 2018. Avances en el estudio de los Coleópteros del Neotrópico. pp. 1-7. En: Deloya, C.; Gasca Álvarez, H. J. (Eds). Escarabajos del Neotrópico (Insecta: Coleoptera). S y G editores, México, 264 p.

EGGLETON, P. 2020. The state of the world's insects. Annual Review of Environment and Resources 45 (1): 61-82. https://doi. org/10.1146/annurev-environ-012420-050035

ETTER A., W. VAN WYNGAARDEN. 2000. Patterns of landscape transformation in Colombia, with emphasis in the Andean region. Ambio 29 (7): 432-439. https://doi.org/10.1579/00447447-29.7.432

GARCÍA-ROBLEDO, C.; KUPREWICZ, E.K.; BAER, C.S.; CLIFTON, E.; HERNÁNDEZ, G. G.; WAGNER, D. L. 2020. The Erwin equation of biodiversity: From little steps to quantum leaps in the discovery of tropical insect diversity. Biotropica 52 (4): 590-597. https://doi.org/10.1111/btp.12811.

GARCÍA, K.; BOTERO, J. P.; MARTÍNEZ, N. J. 2019. New species and new geographical records in South American Piezocerini (Coleoptera: Cerambycidae) with revised keys to species of Alienosternus and Hemilissa. The Canadian Entomologist 1-18. https://doi.org/10.4039/tce.2019.68.

GASCA-ÁLVAREZ, H. J.; AMAT-GARCÍA, G. 2010. Synopsis and key to the genera of Dynastinae (Coleoptera, Scarabaeoidea, Scarabaeidae) of Colombia. ZooKeys 34: 153-192. https://doi. org/10.3897/zookeys.34.309

GIRÓN, J. C.; CARDONA-DUQUE, J. 2018. Estado del conocimiento de los Curculionidae en Colombia. pp. 167-199. En: Deloya, C.; Gasca Álvarez, H. J. (Eds), Escarabajos del Neotrópico (Insecta: Coleoptera). $\mathrm{S}$ y $\mathrm{G}$ editores, México, $264 \mathrm{p}$.

GONZÁLEZ-CÓRDOBA, M.; ZUÑIGA, M DEL C.; MANZO, V. 2002. La familia Elmidae (Insecta: Coleoptera: Byrrhoidea) en
Colombia: riqueza taxonómica y distribución. Revista de la Academia Colombiana de Ciencias Exactas Físicas y Naturales 44 (171): 522-553. https://doi.org/10.18257/raccefyn.1062

GONZÁLEZ-CÓRDOBA, M.; ZÚÑIGA, M. DEL C.; MANZO, V.; GRANADOS-MARTÍNEZ, C. PANCHE, J. 2019. Nuevos registros y datos de distribución de diez especies y cuatro géneros de élmidos (Coleoptera: Elmidae) para Colombia. Boletín Científico del Centro de Museos del Museo de Historia Natural de la Universidad de Caldas 23(1): 247-266. http://www.scielo.org. co/scielo.php?pid=S0123-30682019000100247\&script $=$ sci_ abstract\&tlng=en

GONZÁLEZ-RODRÍGUEZ, L. M;; GARCÍA-HERNÁNDEZ A. L.; CLARKSON, B. 2017. First records of water scavenger beetle species (Coleoptera, Hydrophilidae) from Quindío Department, Colombia. Check List 13 (5): 605-620. https://doi. org/10.15560/13.5.605

GRIMALDI, D.; ENGEL, M. S. 2005. Evolution of the Insects. Cambridge University Press, New York and Cambridge. 755 p.

HOWDEN, A. T. 1976. Pandeleteius of Venezuela and Colombia (Curculionidae: Brachyderinae: Tanymecini). Memoirs of the American Entomological Institute 24: 1-310.

JIMÉNEZ-FERBANS, L.; AMAT-GARCÍA, G.; REYES-CASTILLO, P. 2018. Estudios de los pasálidos (Coleoptera: Passalidae) de Colombia. pp. 81-94. En: Deloya, C. \& Gasca Álvarez, H. J. (Eds), Escarabajos del Neotrópico (Insecta: Coleoptera). S y G editores, México, $264 \mathrm{p}$.

LADINO, A. G. 2019. Lista de Lampyridae del Museo Entomológico Francisco Luis Gallego - MEFLG. Boletín del Museo Entomológico Francisco Luis Gallego 11 (3): 5-22. https:// ciencias.medellin.unal.edu.co/museos/entomologico/images/ Boletin/2019-09/Boletn_Museo_V11_N3_17_Feb.pdf

MARSHALL, S. A. 2018 Beetles: the natural history and diversity of Coleoptera. Firefly Books (US) Incorporated. 784 pp.

MARTÍNEZ, C. 2000. Escarabajos longicornios (Coleoptera: Cerambycidae) de Colombia. Biota Colombiana 1 (1): 76105. http://revistas.humboldt.org.co/index.php/biota/article/ view/57/57

MARTÍNEZ, C. 2005. Introducción a los escarabajos Carabidae (Coleoptera) de Colombia. Instituto de Investigación de Recursos Biológicos Alexander von Humboldt. Bogotá D. C., Colombia. 546 p. http://repository.humboldt.org.co/handle/20.500.11761/9488

MARTÍNEZ-REVELO, D.; MEDINA C. 2017. Diagnóstico de la colección de escarabajos coprófagos (Scarabeidae: Scarabaeinae) del Museo Entomológico Francisco Luis Gallego de la Universidad Nacional de Colombia (sede Medellín). Revista de la Facultad de Ciencias 6 (1): 93-106. https://revistas.unal.edu.co/ index.php/rfc/article/view/62794

MARTÍNEZ-REVELO, D. E.; LOPERA-TORO, A.; MEDINA, C. A. 2020. Review of Scatimus Erichson (Coleoptera: Scarabaeidae) in Colombia with the description of a new species. Zootaxa 4890 (4): 521-534. https://doi.org/10.11646/zootaxa.4890.4.5

MITTERMEIER, R. A.; GOETTSCH, C. 1997. Megadiversidad. Los países biológicamente más ricos del mundo. Cemex, Ciudad de México. 502 p.

MEDINA, C. A.; LOPERA-TORO, A.; VÍTOLO, A.; GILL, B. 2001. Escarabajos coprófagos (Coleoptera: Scarabaeidae: Scarabaeinae) de Colombia. Biota Colombiana 2 (2): 131-144. http:// revistas.humboldt.org.co/index.php/biota/article/view/95

MONNÉ M. A.; CHABOO, C. S. 2015. Beetles (Coleoptera) of Peru: A Survey of the Families. Cerambycidae, Disteniidae, Vesperidae. Journal of the Kansas Entomological Society 88 (1): 34120. https://doi.org/10.2317/JKES1410.13.1

MONNÉ, M. L.; COSTA, C. 2020. Coleoptera in Catálogo Taxonômico da Fauna do Brasil. PNUD. Disponible en: http://fauna. jbrj.gov.br/fauna/faunadobrasil/223. [Fecha revisión: 1 diciembre 2020)

MORENO, L. A.; ANDRADE, G. I.; Y RUÍZ-CONTRERAS, L. F. (Eds.). 2016. Biodiversidad 2016. Estado y tendencias de la biodiversidad continental de Colombia. Instituto de Investigación 
de Recursos Biológicos Alexander von Humboldt. Bogotá, D. C., Colombia. 106 p.

NEITA-MORENO, J. C; GAIGL, A. 2008. Escarabajos de importancia agrícola en Colombia (Coleoptera: Scarabaeidae «Pleurosticti»). Bogotá: Universidad Nacional de Colombia, Produmedios; $161 \mathrm{p}$.

NEWTON, A. F.; GUITIÉRREZ-CHACON, C.; CHANDLER, D. S. 2005. Checklist of the Staphylinidae (Coleoptera) of Colombia. Biota Colombiana 6 (1): 1-72. http://revistas.humboldt.org. co/index.php/biota/article/view/148

OCAMPO, F. C.; BALLERIO, A. 2006. Phylogenetic analysis of the scarab family Hybosoridae and monographic revision of the New World subfamily Anaidinae (Coleoptera: Scarabaeoidea). 4. Catalog of the subfamilies Anaidinae, Ceratocanthinae, Hybosorinae, Liparochrinae, and Pachyplectrinae (Scarabaeoidea: Hybosoridae). Bulletin of the University of Nebraska State Museum 19: 178-209. https://digitalcommons.unl.edu/museumbulletin/2/

PARDO-LOCARNO, L. C.; SCHOOLMEESTERS, P. 2019. Small dung beetles of Colombia (Coleoptera Scarabaeoidea Aphodiinae) I: Preliminary Catalog and Key for Registered Species. Boletín Científico Museo de Historia Natural de la Universidad de Caldas 23 (1): 279-302. http://boletincientifico.ucaldas.edu. co/downloads/Boletin(23)1_13.pdf

RAFAEL, J.A.; MELO, G. A. R.; CARVALHO, C. J. B.; CASARI, S. A.; CONSTANTINO, R. 2012. Insetos do Brasil Diversidade e Taxonomia. Holos Editora, Ribeirão Preto. 795 p.

ROMERO M.; CABRERA E.; ORTIZ N. 2008. Informe sobre el estado de la biodiversidad en Colombia 2006-2007. Instituto de Investigación de Recursos Biológicos Alexander von Humboldt. Bogotá D. C., Colombia. 181 p.

SCHOLTZ, C. H. 1990. Revision of the Trogidae of South America (Coleoptera: Scarabaeoidea). Journal of Natural History 24 (6): 1391-1456. https://doi.org/10.1080/00222939000770841

SIB COLOMBIA. 2020. Biodiversidad en Cifras 2020. https:// sibcolombia.net/biodiversidad-en-cifras-2020/ [Fecha revisión: 1 diciembre 2020)

TABARES-DUQUE, M. E.; QUIROZ-GAMBOA, J. A.; GUARIN-CANDAMIL, G. DE J. 2016. Lista de Lucanidae del MEFLG. Boletín del museo entomológico Francisco Luis Gallego 8 (1): 19-21. https://ciencias.medellin.unal.edu.co/ museos/entomologico/images/Boletin/2016-01/BOLETINFINAL-15-DE-JULIO--19-21.pdf

VITOLO, A. L.; PEARSON, D. L. 2003. Escarabajos tigre (Coleoptera: Cicindelidae) de Colombia. Biota Colombiana 4 (2): 167-174. http://revistas.humboldt.org.co/index.php/biota/article/ view/131

\section{Contribuciones de los autores}

Jennifer C. Girón: conceptualización, curaduría de datos, escritura (borrador original), escritura (correcciones del arbitraje y de edición); Germán Amat-García: conceptualización, escritura (borrador original); Juan Pablo Botero: conceptualización, curaduría de datos, escritura (borrador original), escritura (correcciones del arbitraje y de edición); Juliana Cardona-Duque: conceptualización, curaduría de datos, escritura (borrador original), escritura (correcciones del arbitraje y de edición); Julián Clavijo-Bustos curaduría de datos, escritura (borrador original); Camila Díaz-Durán: escritura (borrador original); Jorge Humberto García Concha: escritura (borrador original); Kimberly García: curaduría de datos, escritura (borrador original), escritura (correcciones del arbitraje y de edición); Wilber López Murcia: escritura (borrador original); Andrés Lugo de la Hortúa: escritura (borrador original); Jhon Cesar Neita Moreno: escritura (borrador original), escritura (correcciones del arbitraje y de edición); José M. Ramírez-Salamanca: curaduría de datos, escritura (borrador original), escritura (correcciones del arbitraje y de edición); Carlos Taboada-Verona: escritura (borrador original); Diego Uchima Taborda: escritura (borrador original); Alejandra Viasus-Bastidas: escritura (borrador original).

\section{Conflictos de interés}

Los autores declaramos no tener conflictos de intereses. 